of which $20 \%$ is wet AMD forces us to consider the need to revise our opinion of the sustainability of the treatment of the disease.

No conflict of interest.

\section{DSL-016 HOW WOULD PHYSICIANS AND NURSES HANDLE THE PROBLEM OF DRUG SHORTAGES?}

doi:10.1136/ejhpharm-2013-000276.259

B Laszloffy. SMZ-Süd Kaiser Franz Josef Spital, pharmacy, Vienna, Austria

Background We have all experienced drug shortages for different reasons, such as licence withdrawals, lack of raw materials, etc. Following internal suggestions in cooperation with the Vienna Health Association an alteration list of drug changes was introduced as a standard process. This chart is updated daily and is posted on the opening intranet website of a 720-bed hospital.

Purpose To find out via a survey how health care professionals are affected by such drug supply interruptions, what ideas they have to reduce the harm to their patients, what suggestions and management they expect from the pharmacy and the manufacturer.

Materials and Methods This survey was done on different wards covering the following aspects:

- recognition level/benefit of the up-to-date drug changes list

- use/knowledge of various pharmaceutical services

- requests/solutions in the recurrent cases of certain drug shortages in our hospital.

Results 77 people (23 physicians and 54 nurses) answered the survey. Half of them were conscious of varying drug shortages (rating scale $0-5$ ) being a worldwide problem. Only 50\% recognised the data provided on the hospital in-house homepage.

The survey focused on proposals to cope with missing drugs. It noted two essential categories:

- importance of pharmaceutical services on the wards

- logistics: the responsibility manufacturers and the pharmacy to immediately inform them of drug shortages, optimal cooperation with other health care providers

- cooperation and teaching of the patient

Conclusions The ward staff are not at all aware of the worldwide drug shortages. The positive impact of the clinical pharmacy service was mentioned by nearly everyone.

No conflict of interest.

\section{DSL-017 IMPORTING MEDICINES - REVIEW OF FIVE YEARS IN A PORTUGUESE HOSPITAL}

doi:10.1136/ejhpharm-2013-000276.260

C Sousa. Hospital de Faro EPE, Pharmacy, Faro, Portugal

Background The pharmaceutical market has always faced several constraints over the years. Nowadays, more than ever, drug shortages have reached critical levels in European countries. Importing medicines usually implies more paper work, different logistics, higher pricing and higher storage costs. Shortages occur not only because the medicine may be sold out but also because there is no marketing authorization. This may affect potentially all drug classes, raw materials and medical devices. Importation procedures in Portugal require annual authorization from the National Medicines' Agency. Rational and cost-effective procurement of medicines in foreign countries can be a challenge for hospital pharmacists.

Purpose The objective is to analyse, classify and evaluate the consumption and costs of medicines imported over the last five years.

Materials and Methods Retrospective analysis of the importation processes and records of imported medicines during the period of 2007 to 2012 .
Results During the period of study 577 importation processes were developed (115 processes a year). About $80 \%$ of these medicines are recommended by the Portuguese National Hospital Pharmacy Formulary and the drug classes most involved were central nervous system, antimicrobial and cardiovascular drugs. The mean price per unit rose $1.2 \%$ since 2007 . In 2012 suppliers are mainly industry/manufacturers while in 2007 there were wholesalers and legal representatives in Portugal. The process of procurement and regulatory issues regarding the importation takes about 14 pharmaceutical hours/week.

Conclusions Importation of medicines at the hospital level is today more often part of the daily tasks of pharmacists. Because the legal framework and logistics are different it is helpful if pharmacists have experience in this matter. The increase in the mean pricing of only $1.2 \%$ was possible specifically because intermediates in the supply chain were reduced.

No conflict of interest.

\section{DSL-018 OPTIMIZATION OF A DRUG REPACKAGING AREA THROUGH THE DEVELOPMENT OF A PROTOCOL IN A TERTIARY HOSPITAL}

doi:10.1136/ejhpharm-2013-000276.261

M Ferrit, M Alvarez, P Aznarte, A Madrid, E Puerta, N Martinez, MA Calleja. University Hospital Virgen de las Nieves, Pharmacy, Granada, Spain

Background The Pharmacy Service includes a unit dose medicines dispensing section. Drug repackaging consists of repackaging drugs which are not within the unit dose system. This process consumes much of the time of the pharmacy technician.

Purpose To establish a working protocol in the repackaging section and measure the work done in the area after the establishment thereof.

Materials and Methods We developed and distributed working protocols. Later, we distributed them to the technical personnel working exclusively in the repackaging area. We performed a prospective observational study (2011). The variables were: repackaging volume (total drugs repackaged, repackaged tablets/month, repackaged tablets/year), repackaging time (total repacking time, lighting and heating time of the repackaging and cutting machine, repackaging and annotation time) and classification of drugs according to the expiry date.

Results The repackaging process steps set out in the protocol were: lighting the repackaging machine, medicines preparation, cleaning of the repackaging area. Completion of the quality control repackaging sheet. Repackaged drugs must be fully identified. The total volume of the repackaged drugs was 300, 39,498 tablets/ month, 479,979 tablets/year, and the time devoted to packaging: cutting time 2 seconds, heating time of 2 seconds, cutting time $1 \mathrm{~min} / 12$ blisters, repackaging time $8.5 \mathrm{~min} / 120$ packs. $24 \%$ of the drugs had an expiry $>3$ years.

Conclusions Repackaging is $25 \%$ of the workload of the pharmacy technicians. The new system enables the staff to work more efficiently, decreasing the repackaging time with a high volume of drugs repackaged/year. The expiry date of the repackaged drugs must be extended in order to obtain a better use of resources.

No conflict of interest.

\section{DSL-019 OPTIMIZATION OF INFLIXIMAB USE CAN SAVE MONEY}

doi:10.1136/ejhpharm-2013-000276.262

${ }^{1} \mathrm{C}$ Cuesta-Grueso, ${ }^{1} \mathrm{JE}$ Poquet-Jornet, ${ }^{2} \mathrm{C}$ Santos-Ramirez, ${ }^{3 \mathrm{~J}}$ Estelles-Arnau. ${ }^{1}$ Hospital de Denia, Pharmacy, Denia, Spain; ${ }^{2}$ Hospital de Denia, Rheumatology, Denia, Spain; ${ }^{3}$ Hospital de Denia, Digestive Medicine, Denia, Spain

Background Intravenous mixtures with low physicochemical stability vials could generate economic loss by wasted medication in 